\title{
Determination of Levels of IgE and IgG and the Activity of Complement Comparatively in Blood and Lymph in the Experimental Arthus and Overy Phenomenon
}

\author{
Aliyeva Tarana Rzakuli \\ Department of Pathological Physiology, Azerbaijan Medical University, Azerbaijan, Baku \\ Email address: \\ tarana-aliyeva-2016@mail.ru \\ To cite this article: \\ Aliyeva Tarana Rzakuli. Determination of Levels of IgE and IgG and the Activity of Complement Comparatively in Blood and Lymph in the \\ Experimental Arthus and Overy Phenomenon. International Journal of Immunology. Vol. 7, No. 3, 2019, pp. 37-40. \\ doi: $10.11648 /$ j.iji.20190703.12
}

Received: July 4, 2019; Accepted: September 12, 2019; Published: September 27, 2019

\begin{abstract}
The aim of investigation was the determination of the levels of IgE, IgG and complement activity in the blood and lymph in the experimental Arthus and Overy phenomenon. Experiments were carried out on 27 chinchilla rabbits (weight 2.5$3 \mathrm{~kg}$ ) in three series. To reproduce the Arthus phenomenon, rabbits were sensitized by subcutaneous administration of $1 \mathrm{ml}$ of horse serum to the scapular region every 5 days; after the fifth injection in the injection area of horse serum the necrosis was observed. To obtain the Overy phenomenon, a resolving dose of horse serum was injected subcutaneously in a sensitized animal, and an Evans blue was introduced into the auricular vein. In this case, the injection area is colored blue. The blood necessary for the experiment was taken from the marginal auricular vein of the rabbit, and lymph - from the thoracic lymphatic duct. The levels of IgE and IgG and the activity of the complement comparatively in the blood and lymph in the experimental Arthus and Overy phenomenon were studied. The experiments were carried out on 27 rabbits of the «Chinchilla» breed in two series. The studied parameters of IgE and IgG and complement activity in blood and lymph of intact animals served as control. The results of the study showed that the level of IgG in the Arthus phenomenon increases and IgE decreases. While at the Overy phenomenon, on the contrary, the level of IgE increases and the level of IgG decreases. The titer of complement in immune complex reactions is sharply reduced; it indicates the involvement of complement with IgG elimination of the allergen.
\end{abstract}

Keywords: Immunoglobulins, Arthus and Overy Phenomenon, Complement

\section{Introduction}

IgE plays a key role in the development of allergic diseases of immediate type [2]. The increase in the level of interleukin $4\left(\mathrm{IL}_{4}\right)$ in atopic reactions leads to an increase in the secretion of IgE antibodies and the connection of a cascade of IgE-mediated allergic reactions involving mast cells, basophils and eosinophils. Studies have shown that the several isotype antibodies are synthesized during the immune responses, most of which are IgG antibodies [3]. It is shown that the induction of $\mathrm{IgG}$ antibodies to the main allergen reduces the production of $\operatorname{IgE}$. And also, there is an opinion, in connection with reagins not only with $\operatorname{IgE}$, but also with IgG subclasses. The key role in the mechanisms of immunological recognition of foreign antigens is played by antibodies of G3 subclasses, which in the process of immune response connect a cascade of mechanisms leading to the destruction and elimination of foreign antigens and pathogens [5]. And G4 antibodies are involved in the mechanisms of allergic inflammation, but do not provide for the destruction and elimination of allergens and atopenes from the body [1].

Evidence suggests that the complement system may affect the course of many immune processes [5]. The change in the complement profile indicates the existence of its different types, confirms the activation of the complement cascade with signs of classical and alternative pathways. The alternative pathway has a low level of basic activity, so it requires control by regulatory molecules. In case of violation of these regulatory molecules on the cell membranes, the 
complement amplification accompanied by an increase in the synthesis of proinflammatory mediators such as $\mathrm{C} 3 \mathrm{~A}$ and C5A, which carry out dynamic regulation of the immune response, occurs [13]. They (mainly C5A) cause accumulation and activation of polymorph-nuclear leukocytes, leading to the release of lysosomal enzymes and peroxidation products, reduction of smooth muscle cells, histamine release from mast cells and increase the vascular permeability [3].

There are the number of regulators (e.g. factor $\mathrm{H}$ ) controlling the complement. In the absence of sufficient binding of $\mathrm{H}$ factor and its protective properties of the tissue may be exposed to complement, resulting in the formation of immune and inflammatory reactions. Some authors note the correlation between the content of CIC in the blood, the complement system and the severity of atopic diseases [12].

Given the above, we conducted this study, which aims to determine the levels of $\operatorname{IgE}, \operatorname{IgG}$ and complement activity in the blood and lymph in the experimental Arthus and Overy phenomenon.

\section{Materials and Methods}

Experiments were carried out on 27 chinchilla rabbits (weight $2.5-3 \mathrm{~kg}$ ) in three series. The investigated indicators of $\mathrm{IgE}$ and $\mathrm{IgG}$, and complement in blood and lymph of intact rabbits (9 rabbits) served as control. In the first series (9 rabbits) these indicators are identified on the model of the Arthus phenomenon, in the second ( 9 rabbits) - on the model of the Overy phenomenon.

To reproduce the Arthus phenomenon, rabbits were sensitized by subcutaneous administration of $1 \mathrm{ml}$ of horse serum to the scapular region every 5 days; after the fifth injection in the injection area of horse serum the necrosis was observed. To obtain the Overy phenomenon, a resolving dose of horse serum was injected subcutaneously in a sensitized animal, and an Evans blue was introduced into the auricular vein. In this case, the injection area is colored blue.

The blood necessary for the experiment was taken from the marginal auricular vein of the rabbit, and lymph - from the thoracic lymphatic duct by the method of A. A. Kornienko in the modification of M. Kh. Aliyev and V. M. Mammadov [10]. Determination of the level of IgE and IgG in the blood and lymph of animals was performed by solidphase immune-enzyme analyzer (SpIEA) on the appropriate test systems ("Farmingen", USA). The results of the study were evaluated on a semi-automatic analyzer Stat-Fax-2000 (USA). Determination of complement activity in the blood and lymph were carried out according to the method of L. S. Reznikova [11].

The methods of descriptive statistics, Wilcoxon-MannWhitney rank criterion were applied in the statistical processing of the obtained data. The average value of the obtained samples was applied in the format $\mathrm{M} \pm \mathrm{m}$ (min$\max )$.

\section{Results}

As a result of our study, it was found that the determination of $\mathrm{IgE}$ and $\mathrm{IgG}$ in the experimental Arthus and Overy phenomenon revealed their multidirectional change. Thus, both at the stage of sensitization and at the stage of resolution of the Arthus phenomenon, there was an increase in the level of $\operatorname{IgG}$ and a decrease in the level of $\operatorname{IgE}$, but with the Overy phenomenon, on the contrary, increased the level of IgE, decreased IgG. The titer of the complement was reduced as in the Arthus phenomenon, and the phenomenon of Overy, but the decline in this indicator with the Arthus phenomenon was more significant (table 1).

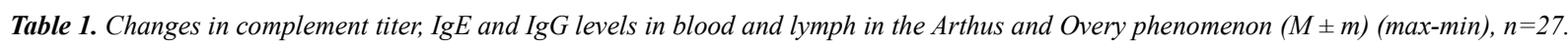

\begin{tabular}{|c|c|c|c|c|}
\hline \multirow[t]{3}{*}{ Indicators } & \multicolumn{4}{|c|}{ Phenomenon Arthus (n=9) } \\
\hline & In blood & & In lymph & \\
\hline & 5th day of sensitization & 25th day of sensitization & 5th day of sensitization & 25 th day of sensitization \\
\hline Complement (h.u.) & $36,4 \pm 0,3^{*}(37,8-34,6)$ & $4,8 \pm 0,2^{*}(6-4)$ & $20,7 \pm 0,3^{*}(23,1-19,8)$ & $3,8 \pm 0,4^{*}(5,4-1,8)$ \\
\hline $\mathrm{IgE}, \mathrm{mE} / \mathrm{L}$ & $19,11 \pm 1,12^{\wedge}(24-14)$ & $13,58 \pm 0,40 *(15-12)$ & $8,05 \pm 0,38^{\wedge}(9,5-6,2)$ & $6,32 \pm 0,33 *(7-5)$ \\
\hline $\operatorname{IgG} g / 1$ & $9,46 \pm 0,24(10,5-8,5)$ & $23,13 \pm 0,72(26-20)$ & $4,14 \pm 0,27(5-2,9)$ & $12,07 \pm 1,04(14-8,8)$ \\
\hline \multicolumn{5}{|c|}{ Phenomenon Overy $(n=9)$} \\
\hline & In blood & & In lymph & \\
\hline & 6th day of sensitization & 12th day of sensitization & 6th day of sensitization & 12th day of sensitization \\
\hline Complement (h.u.) & $27,4 \pm 0,4^{*}(30,1-25,9)$ & $11,3 \pm 0,4 *(13,8-9,9)$ & $22,6 \pm 0,3^{*}(24-20,9)$ & $10,4 \pm 0,8^{*}(14,4-6,9)$ \\
\hline $\mathrm{IgE}, \mathrm{mE} / \mathrm{L}$ & $12,56 \pm 0,63 \#(10-16)$ & $16,90 \pm 0,41^{*}(15,6-19)$ & $5,54 \pm 0,32 *(4,7-7,2)$ & $8,5 \pm 0,36 *(7-10)$ \\
\hline $\mathrm{IgG} g / 1$ & $6,8 \pm 0,29^{\wedge}(4,9-7,8)$ & $9,7 \pm 0,92 *(6,6-13,8)$ & $3,93 \pm 0,33 *(2,6-4,7)$ & $4,57 \pm 0,51^{\wedge}(3-7)$ \\
\hline \multicolumn{5}{|l|}{ Intact animals $(n=9)$} \\
\hline & In blood & & In lymph & \\
\hline Complement (h.u.) & $41,1 \pm 0,7(45-38)$ & & $31,2 \pm 0,5(33-29,1)$ & \\
\hline $\mathrm{IgE}, \mathrm{mE} / \mathrm{L}$ & $10,26 \pm 0,40(12-8)$ & & $4,48 \pm 0,35(6-3)$ & \\
\hline $\mathrm{IgG} \mathrm{g} / 1$ & $5,39 \pm 0,53(8-3)$ & & $2,92 \pm 0,13(3,5-2,3)$ & \\
\hline
\end{tabular}

Note: reliable interval in comparison with the intact group animals at: ${ }^{*} \mathrm{p}<0.001 ;{ }^{\wedge} \mathrm{p}<0.05 ; \# \mathrm{p}<0.01$.

With the Arthus phenomenon, the level of IgG in the blood of animals is increased, while $\operatorname{IgE}$ - decreased. Thus, in the period of sensitization ( 5 th day), the level of IgE in the blood is increased 1.8 times, and the level of $\operatorname{IgG}-2.0$ times $(\mathrm{p}<0.001)$ compared with intact animals and amounted to $5.86 \mathrm{~g} / \mathrm{l}$. On the 25th day of the Arthus phenomenon, the level of $\operatorname{IgE}$ in the blood is increased 1.4 times compared to intact animals and was $13.58 \pm 0.40 \mathrm{mE} / 1(\mathrm{p}<0.001)$. The IgG 
level is increased 4.1times and amounted to $23.13 \mathrm{~g} / 1 \pm 0.72$.

If in animals with the reproduced Arthus phenomenon at the stage of sensitization ( $5^{\text {th }}$ day) the complement titer in blood is decreased in comparison with intact animals by 1.1 times and was equal to $36.4 \pm 0.3 \mathrm{~h}$.u., but in the resolving period the complement titer is sharply decreased and amounted to $4.8 \pm 0.2 \mathrm{~h}$.u.

In the Overy phenomenon, related to skin-anaphylactic reactions, levels of immunoglobulins were changed as follows: during the period of sensitization of Overy phenomenon the IgE level was increased 1.2 times, and in animals with the reproduced Overy phenomenon, 1.6 times as compared with intact animals. The level of IgG in the period of sensitization increased 1.3 times $(p<0.05)$, compared with the corresponding indicator in intact animals, whereas in the phenomenon of Overy this increase was 1.8 times more than in intact animals $(p<0.001$ If in the period of sensitization of the Overy phenomenon, the complement titer in the blood decreases by 1.5 times compared to intact animals, it was equal to 27.4 h.u., in animals with the reproduced Overy phenomenon the complement titer in the blood decreasing 3.6 times compared to intact animals was equal to $11.30 \mathrm{~h}$.u.

Compared with peripheral blood in the lymph there was a decrease in the studied parameters.

In lymph at the Arthus phenomenon on the 5th day of sensitization the levels of $\operatorname{IgE}$ and $\operatorname{IgG}$ were increased in comparison with intact animals in 1,2 and 2,3 times, the complement titer in this period in comparison with indicators in intact rabbits is increased in 1,1 times $(p<0,001)$. On the 25 th day of sensitization (in the period of Arthus phenomenon) IgE level is increased in comparison with intact animals by 1.6 and $\operatorname{IgG} 5.0$ times $(\mathrm{p}<0.001)$ and amounted to $6.32 \mathrm{mE} / \mathrm{l} \pm 0.33$ and $12.07 \mathrm{~g} / \mathrm{l} \pm 1.04$ respectively.

On the 6th day of sensitization of the Overy phenomenon the levels of $\mathrm{IgE}$ and $\mathrm{IgG}$ in lymph are increased by 1.2 and 1.3 times, respectively, compared with intact animals, then at the reproduced Overy phenomenon the levels of $\mathrm{IgE}$ and $\mathrm{IgG}$, increasing by 1.9 and 1.6 times compared with the indicators in intact animals, amounted to $8.5 \mathrm{mE} / 1 \quad(\mathrm{p}<0.001)$ and $4.6 \mathrm{~g} / 1$ $(p<0.01)$, respectively. At this time, the complement titer is decreased by 2.18 times in comparison with intact animals.

At the stage of sensitization in experimental animals at the injection site, hyperemia followed by the development of infiltration was noted. Further, during the formation of the hyperergic inflammation focus (20-25th days of sensitization), tissue necrosis was observed at the injection site in experimental animals. In some animals, necrosis occupied a large area of the site, while others showed only hyperemia without necrosis. All animals showed an increase in blood clotting, both in the period of sensitization and in the period of shock.

In 4 animals with a replicated Overy phenomenon, skin coloration with the Evans blue was weak, and in 5 animals it was more pronounced.

\section{Discussions}

Some researchers observed low rates of the CIC which correlated with a high titer of $\mathrm{C} 4$ component of complement and the increased concentration of $\operatorname{IgE}$ in serum for the course of atopic allergic diseases $[9,11,12]$. Others note that even in such pathological conditions as parasitic infestations, Wiskott-Aldrich syndrome, the concentration of serum $\operatorname{IgE}$ is several thousand times lower than the titer of serum IgG.

In our studies, an increase in the level of $\operatorname{IgE}$ and a decrease in IgG are observed in the Overy phenomenon, while in the Arthus phenomenon, on the contrary, the level of IgG increases, and IgE decreases. An increase in IgG leads to a significant decrease in the titer of complement during the Arthus phenomenon. An increase in the level of $\operatorname{IgE}$ with the Overy phenomenon leads to minor changes in the titer of complement in comparison with the Arthus phenomenon.

\section{Conclusions}

The results of our studies have shown that the level of IgG increases in the Arthus phenomenon, while $\operatorname{IgE}$ decreases. Increased IgG and decreased IgE levels lead to suppression of allergy symptoms. In this case, the symptoms disappear gradually. And with the Overy phenomenon, on the contrary, against the background of a decrease in $\mathrm{IgG}$, the level of $\operatorname{IgE}$ increases and allergy symptoms increase slightly. This indicates that immunoglobulin $G$ slightly inhibits allergic reactions. The results of the study showed that in immunocomplex reactions the complement titer decreases sharply. This indicates the participation of the complement in the elimination of the allergen. It also becomes clear that complement is involved in allergen elimination, along with IgG. As a result of studies it was found that changes in these parameters in the blood are more significant than in the lymph.

\section{References}

[1] Babakhin A. A., Shilovsky I. P., Andreev I. V., Cozmin A. D. et al. Experimental allergen-specific immunotherapy of timpol allergo-vaccine on the model of IgE-dependent bronchial asthma in mice//Immunology, 2012, №3, pp. 134-142.

[2] Gushin I. C. IgE-mediated hypersensitivity as a response to the barrier function of tissues//Immunology, 2015, Vol. 36, no. 1, pp. 45-52.

[3] Konisheva A. Y., Samoylikov P. A., Shternshis Y. A., Gervazieva V. B. Determination of autoantibodies of antibodies to tissue antigens in patients with allergic diseases//Immunology, 2010, vol. 11.№3, p. 17-22.

[4] Kuznik B. I. Coagulability and fibrinolytic activity of lymph in various pathological conditions//Pathological physiology and experimental therapy, 2013 №1, p. 39-42.

[5] Ukhanova O. P. Study of the effect of monoclonal antibodies to human IgE on activation of peripheral blood basophils in patients with seasonal allergic rhinitis//Immunology, 2010, vol. 11.№1, p. 34-39. 
[6] Fedicheva N. A. Qorbov V. V. Features of cytokine balance and immunoglobulin $\mathrm{E}$ concentration in patients with chronic nephritis depending on the results of treatment//Medical immunology, 2010, T12, № 4-5, p. 89-91.

[7] Shatokhin M. N., Konoplya A. I., Teodorovich O. V., Karaulov A. V. Cytokine status and complement system in chronic prostatitis. Pharmacological correction of violations//Immunology, 2013, №1, p. 156-159.

[8] Dodge M., Quinata K, Stinson K. Effective work with Excel/English translation. St. Petersburg, 2000; (in Russian).

[9] Klos A., Wende E., Wareham K. J. et al. International Union of Pharmacology Complement peptide C5a, C4a and C3a reseptors. Pharmacol. Rev. 2013; 65: 500-543.
[10] Kornienko A. A., Kulikovskii N. N., Sorokaty A. E. Catheterization of the thoracic duct in the experiment. Topical issues of topographic anatomy and operative surgery. M., 1977; 1, 22-6 (in Russian).

[11] Reznikova L. S. Complement and its significance in immunological reactions: Moscow; 1967.

[12] McHarg S., Clark S. J., Day A. J. et al. Age-related macular degeneration and the role of the complement system. Molecular Immunology, 2015, 02. 032.

[13] Silva A. S., Teixeria A. G., Bavia L. et al. Plasma levels of complement proteins from the alternative pathway in patients with age-related macular degeneration are independent of Complement Factor H tyr (4) (0) (2) His. polymorphism. Mol. Vis. 2012; 18: 2288-2299. 